\title{
Study on Etymology Features of Chinese Language from the Ancient Words of Gan Dialect ${ }^{1}$
}

\author{
Jiugen Xiao ${ }^{1, a^{*}}$,Ying Zhou ${ }^{2, b}$ \\ (Jiangxi Normal University Language and Language Life Research Center, Nanchang, China) \\ Email:a 2389549534@qq.com; b992963813@qq.com \\ *Jiugen Xiao
}

Key words: Chinese dialects; Gan dialect; Etymology features

\begin{abstract}
The history of language development tells people that no matter the language or dialect has any problem on the language origin, and it will show different characteristics in the development of origin. According to the etymology of Chinese word, the origin and development have experienced three historical stages, such as the origin, derivation and syn-thesis. So does the Chinese dialect such as Gan dialect. The study of etymology features on Gan dialect not only has a th-eoretical significance but also has practical value for people to further explore its origin, development and related issues.
\end{abstract}

\section{Introduction}

To investigate the origin and development of a language will be related to the etymology. Many concerns about Chi-nese origin and its etymology have been taken by the scholars. At the end of the last century, Mr. Wang Li published Words and Etymology ${ }^{[1]}$, and the result of the study--whether the monographs or the papers are very impressive ${ }^{[2]}$. In recent ten years, there has been a big development of the related research, only the number of the papers is several hundreds, many of which are very important by famous scholars, such as Liu Zhenqian (2011) ${ }^{[3]}$, Wang Mingyu (2012) ${ }^{[4]}$ and Chen Wenjie (2013) ${ }^{[5]}$, etc.. Chinese dialects are concerned without enough attention, especially for Gan dialects (one of Chinese dia-lects in Jiangxi province) which is almost in a state of zero attention in this field.

In the history of Chinese etymology, Mr. Wang Ning once pointed out, "the accumulation of Chinese vocabulary has experienced three stages, that is, the primary stage, the derived stage and the synthesis stage." [6] In fact, the vocabulary development of the Gan dialect has also experienced those stages, and each stage has different characteristics.

According to different characteristics of etymology in Gan dialect, some brief analysis can be made in the following.

\section{Original features of the vocabulary in Gan Dialect}

The original characteristic of the vocabulary refers to the characteristics originated from the original pronunciation and meaning in a language or dialect. The linguistic principle reveals that, in terms of its origin, the biggest characteristic of linguistic signs is that it is arbitrary, and its "speech form and meaning are not necessarily related to each other, only the relationship in social conventions" ${ }^{[7]}$. Because of its long history, people still can not know the original meaning of the vocabulary in ancient times. For example, although there have been fruitful results on the study of Chinese vocabulary, throughout the study of scholars, only from the era of Oracle, before that time, the situation of the original vocabulary is still unknown. To be sure, no matter the language or dialect, they have a lot of original vocabularies. From the history of Chinese vocabulary, the spring and

\footnotetext{
${ }^{1}$ Foundation item: National Social Science Fund Post Funded Projects " Analysis on Origin and Theory of Gan Dialect Archaism "( 14FYY027); Key Projects of Jiangxi Provincial Academy of Social Sciences Joint Planning "Gan Po Dialect and Folk Culture Research" (13YY01)
} 
autumn and Warring States period is a key period for the development of Chinese voca- bulary. In this period, a large number of new words derived from the original vocabulary, in addition, a lot of native words came out, which is a turning point in the development of Chinese vocabulary. The vocabulary in the dialect also develops in this way. In the vocabulary system of Gan dialect, there are many native words from the Pre-Qin period. In terms of the combination of sound and meaning, the initial consonant in the native word retains some ancient sound, $\mathrm{y}$ and $\mathrm{n}$, such as "ai" [ $\left.\mathrm{yai}^{11}\right]$, "ao" [ $\left.\mathrm{yau}^{35}\right]$, "ya" $\left[\mathrm{ya}^{35}\right]$, "ri”" $\left[\mathrm{ni}^{13}\right]$, "ren" $\left[\mathrm{nin}^{213}\right]$, "rou" [niuk $\left.{ }^{5}\right]$. In the native words, light and heavy labials are mixed together, such as "wen" $\left[\mathrm{men}^{35}\right]$, "wang" $\left[\mathrm{mon}^{21}\right]$; some still retain the ancient Rusheng syllable, such as "mu" [muk ${ }^{5}$, "du" [t uk $\left.{ }^{5}\right]$ (Jue syllable), "le" [lok ${ }^{5}$, "shao" [ sok $\left.^{5}\right]($ Le syllable), "bai" $\left[\mathrm{p}^{\prime} \mathrm{ak}^{5}\right]$ (Duo syllable) ${ }^{[8]}$. The phenomenon illustrated in Gan dialect is not entirely due to the inherent law of the combination of the sound and meaning. To a large extent, it should be the function of the "conventional" principle.

Fig.1 The schema of the feature of sound and meaning in original words:

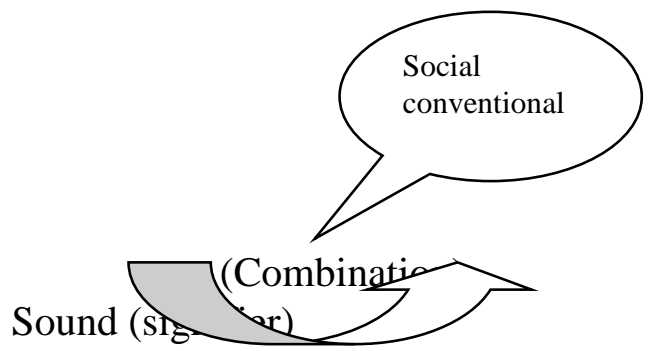

Meaning (signified)

Original words

\section{The derived features of the vocabulary in Gan Dialect}

The derived feature of vocabulary refers to the characteristics of the combination of the sound and meaning in a language or native dialect vocabulary when it derives a new monosyllabic word. From the history of Chinese vocabulary, the Zhou and Qin Dynasties is the peak period of monosyllabic words derived from Chinese original vocabulary, not only many words related with production activities and daily life, also a lot of lexicons with abstract and generality reflecting social ideology, even some function words, which are not common in the past. There is no doubt that, this is also a foun-dation period for the rapid development of the vocabulary of Chinese Gan dialect, which can be seen from the origin of Gan dialect vocabulary.

During this period, when a new Gan word derived from the original word, its features with the combination of the pronunciation and meaning can be shown as follows:

Firstly, several new words with various meanings or pronunciations are derived from one original word, and those derived words are still closely linked together, and the derived words are in the same morphology. For example, the word "zheng" in Gan dialect in Shuowen, Pubu refers to "qi". Duan Yucai notes, "Qi means the wheat grow in the same high." The original meaning seems to refer to "Qiping", which derives "neat and in order" in general. Such as "Zhengju" in the poem in Book of Odes, June, Zheng Xuanjian, "It is very neat with order." In this sense, people in Gan areas such as Fu -zhou, Changdu, Yiliu, Jicha call it "Qizheng", which derives the meaning "governance", such as Dongjing Fu by Zhang Heng in Han dynasty, "zheng the uniform." Xue Zong, "Zheng refers to governance and management." Because of the chaos, things must be reformed by regulation or governance in order to restore the original appearance. In Gan dialect, "zheng (treatment) disease", "zheng (fix up) house", "zheng (mend) car", those "zheng" are closely related with the or-iginal meaning of the word. In Gan dialect, the adjective "zheng" and the verb "zheng" have different 
pronunciation. The former is mostly with word "qi" with light tone in general, for example, "the house is qizheng (neat)", people in Lichuan, the east of Gan pronounce [tcin], in Nanchang, north of Gan pronounce [tsay] or [tsin213], in Jishui, middle of Gan [tin]; the latter, in addition to the tone, the local pronunciations are basically the same, such as Lichuan [tsan44], Nanchang [tsan213], Jishui [tsay213], Pingxiang, western of Gan [tșã35].

Secondly, new meanings are derived from the original word. The new meanings are represented by different pronun-ciations and word shape, but homologous relationship exists between them. Such as "qiao" in Gan dialect, its original meaning is bite. Shuowen, Koubu notes, to bite with teeth. The meaning is commonly used in most areas in Gan dialec, such as "qiao sweet potato", "qiao bones" and so on. The pronunciations are as follows, Lichuan in the east of Gan [ $t$ 'iau13], Nanchang in the north of Gan [t' icu11], Jishui in the middle of Gan [t6' iau31]. The primary meaning derives the meaning of "jiao (chew)", namely repeatedly chewing food. Cangjie, "jiao refers to (chew)." People usually use "jiao" in Changdu, Jicha of Jiangxi. Such as the Pingxiang (a city in Gan) dialect, "it's hard to jiao (chew)." "jiao food for you to eat" As Jishui dialect said, "that piece of meat is really tough, and it is hard to jiao." Its pronunciation is [to' io?2] in Nanchang, [tciau31] in Jishui, [ts' io11] in Pingxiang. In fact, the meaning and usage of "jiao" in Gan dialect inherited from the ancient Chinese, as Huai Nanzi, Linxun notes, by Liu'an in Han dynasty, "jiao food without a good taste." In Gan dialect, "qiao" and "jiao" are often used for abstract meaning, such as "qiao mouth (it is nonsense)", "jiao teeth (make small talk); people from Nanchang also use "jiao in a mess (talk nonsense)", "jiao in blind ((talk nonsense)", "listen to jiao (not believe it)" and so on. Another example, Shuowen, Qianbu, "ai is zi". Duan Yucai explains that, "zi means to scold in original". The original meaning "scold" derives "sigh" or "the sound of sigh". It is shown in Fayan, Yuanqian by Yangxiong in Han dynasty. Li Gui notes, "ai can be a modal particle used in the end of the sentence". It also can be used to answer, such as in Dialect volume 10, so, "people in South Chu usually say 'ai' to express the agreement." However, in the Gan dialect, the expre-ssions of "sigh" and "answer" generally have a clear difference. The expression "sigh" is "[ai53]"; while "[ع53]" is used for answering. An example in Jishui dialect, "ai [ai53], I do not know what the reason for the thing." Another example, "when hearing his father's calling, he ai [ع53], and then went back home." In Gan dialect, "ai" is used for sigh and also exists in the ancient Chinese, Historical Records, Xiang Yu's Biography, "Ai (signing)! They are not worth cooperating." Sima Zhen notes, "ai can be used to express the signing."

Thirdly, a original word can derive many words with etymological meanings or pronunciations. Such as "shao (little)" means "xiao (small)", which derives "miao (the end)", "miao (second)", "miao (small)", "miao (wonderful)". They are the same pronunciation with the different etymological meanings. In Gan dialect, "Shumiao" refers to "the top or the end of the tree". "lu" refers to the meaning of "black", and it derives five different "lu", all of which have the meaning of "black". The first "lu" means yellow and black soil, the second means a black dog in ancient time, the third means black orange, the forth one means black eyes, the last "lu" refers to black. Mr Wang Li said, "the fifth 'lu' and the original word are the same word, although they different in the appearance." [9] At present, people still use the fifth "lu" in Gan dialect, referring to rust iron "sheng lu", which is closely related with the etymological meaning.

Indeed, after Han Dynasty, although the derivation of many new words emerged, they were not the mainstream. The mainstream tends to the polysyllabic synthesis period. When it comes to the monosyllabic words in Things of Poseng, Mr. Yu Liming points out, "from view of formation, the Pre-Qin is the period of peak for the monosyllabic words, after Han dynasty, the tendency is decline, which indicats that the monosyllabic words have not been the new-generated words since then, and the effect of newborn monosyllabic words to future generations also declines." [10] 
Fig.2 Schema on pronunciation and meaning features of original word and its derived words:

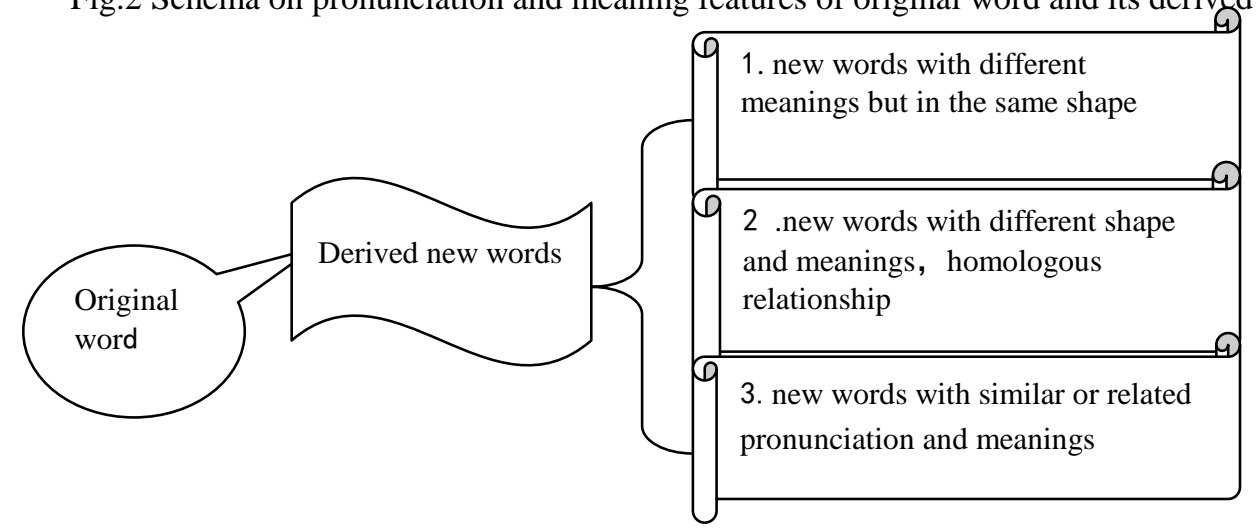

\section{The characteristics of polysyllabic words in Gan Dialect}

In the original and derived stage, the new words are monosyllabic. After a very long period of primary stage and the rapid development of derivation, monosyllabic words have accumulated to a considerable number, which not only bring great burden to people's memory and too much is bound to cause chaos in communication. It is more difficult for people to adapt to the requirement of continuous development in the language expression. Therefore, the synthesis of polysyllabic words is not only the urgent demand on both subject and object, but also the inevitable development of vocabulary.

Then, what is the basic cause for the synthesis of polysyllabic words or disyllablization? Mr. Wang says, "the disy-llablization of Chinese has two main factors: one is the simplification of the pronunciation, the other is the absorption of foreign language... because the disyllablization is one of the important means to reduce the homonym." [11] However, Tang Yuming believes that "these two points are the new factors for the rapid development of polyphony after medieval times, and certainly not the original and basic reason for disyllablization of Chinese". He believes that the source of disyllabli-zation of Chinese is far away in the Shang and Zhou dynasties, and the original motivation is not to reduce the homonym, "but in order to pursue the semantic precision". Regardless of the form of compound words, regardless of the double syllable or multi syllable, all are for the semantic precision, "semantic precision is the basic reason for this. ${ }^{[12]}$

In addition to its basic cause, the compound polysyllabic words also has a prerequisite which is the sufficient amount of monosyllabic words that can be the elements for building the polysyllabic words. Actually, Zhou and Qin dynasties was the heyday of derived monosyllabic words, but polysyllabic words also accounted for a certain proportion, such as the polysyllabic words in Analects of Confucius account for about $15 \%$ of the total, and in the Book of Songs account for approximately the $25 \%{ }^{[13]}$. After Han dynasty, polysyllabic synthesis has become the most important word formation in Chinese. The composition of the Chinese dialect and the vocabulary of the Gan dialect are also generally in the case. In terms of the development of ancient words, in Zhou dynasty and the Qin dynasty, they are mostly monosyllabic words with only a small amount of polysyllabic words; and after Han dynasty, the situation is the opposite. It can be said that, the development of Gan dialect and the common language are basically in synchronous progress.

So, what are the synthetic characteristics of the words? The characteristics are the features of pronunciation, shape and meanings of synthetic words which are formed by several monosyllabic words. The features of polysyllabic words in Gan dialect, especially in the early times, are shown as follows: 
On the one hand, compared to monosyllabic words, the word meaning is more single, clear and precise. A monosy-llabic word usually has the common phenomenon of multi-pronunciation and polysemy, which increases the difficulty in understanding, like the feeling of ambiguous, unnecessary trouble. And polysyllabic word makes the semantic object more clearly, makes the meaning more precise, which greatly reduces the problems in monosyllabic words. In Gan dialect, "fameng" in ancient times is a polysyllabic word combined by "fa" and "meng" with the meaning of "to enlighten the civilization". Yi, Meng, "fameng refers to enlightenment." Its extended meaning "start learning" emerged later in the middle of ancient times. The word "juli" means "make a bow with hands folded in front" or "stand on rituals" since ancient times, until today, the meaning is still used in Gan dialect. The similar archaic words in Gan dialect have "lao'ou (old woman)", "linshe (neighbor)", "qinli (work hard)", "qugui (come back)", "yunti (ladder)" and so on, which all reflect the single, clear and precise meaning.

On the other hand, word building elements for some compound polysyllabic words are not very close. For example, the word form is variable, or word order can be changed, or morpheme synthesis and independence both can be used.

(1) Either interchangeability of Chinese characters or homologous variations build the new words. In Gan dialect, "zengbi" or "zeng'bi", Wang Yun says, "zeng and zeng' are the same, but in similar shape." Guangya, Shiqi, "Zeng refers to zeng'." Wang Niansun points out, "Zeng is the same with zeng'." Qian Yi notes in Dialect, Volume Five, "Zeng and zeng' are the same both in pronunciation and meaning." Another example, "niuzi" refers to "cow". "niuzi" is also called "niuzi"". Guangya, Shishou, "Zhi means female." Wang Niansun, "Zhi means to zi', and a cow refers to 'zi'." The chara -cter "zi" can be the interchangeability of "zi"".

(2) The elements and order of word-forming can be changed, and the meaning does not change. In Gan dialect, "qugui" means "go back home". Spring and Autumn in Wu and Yue, "guiqu refers to go back". So "qugui" is also can be called "guiqu". The two morphemes "qu" and "gui" change the order, but the two meanings do not change. Another exam

-ple, "qizheng" also can be called "zhengqi". Liutao, Longtao, Bingzheng, "the armed forces is qizheng", which also mea-ns "zhengqi". Other examples, "chenhui" and "huichen (dust)", "huangxi" and "xihuang (likeness)", "naore" and "renao (lively)" all belong to the case.

(3) Both single morpheme and compound morpheme can be used. In Gan dialect, the compound word "fameng" in Gaotang $F u$ by Song Yu, "Fameng refers to enlightenment." And the single morpheme "fa" is used in the Analects of Confucius, "Ziyue: "to fa (enlighten) the people who think deeply'." Another one, "jieji" in Gan dialect refers to "taijie". Both "jie" and "ji" have the same meaning. Guangyun, Jieyun, "jie means jieji (class)", and Yupian, Xibu, "ji means jieji (class)". Thus, this case is popular in Gan dialect, which also is common in ancient literatures, such as, Yemiaobei by Lu Guimeng in Tang dynasty, "to improve the jieji (class) can be in a good position." The single morpheme "jie" and "ji" are used in the following examples. Mencius, Lilou, "in the ancient government, people in different jie (classes) do not talk to each other." The book of Rites, Quli, "to clime the ji step by step." These components of the word formation have been combined together for a long time, then gradually become a compound word.

Fig.3 Schema on pronunciation and meaning features of original word and its compound polysyllabic words:

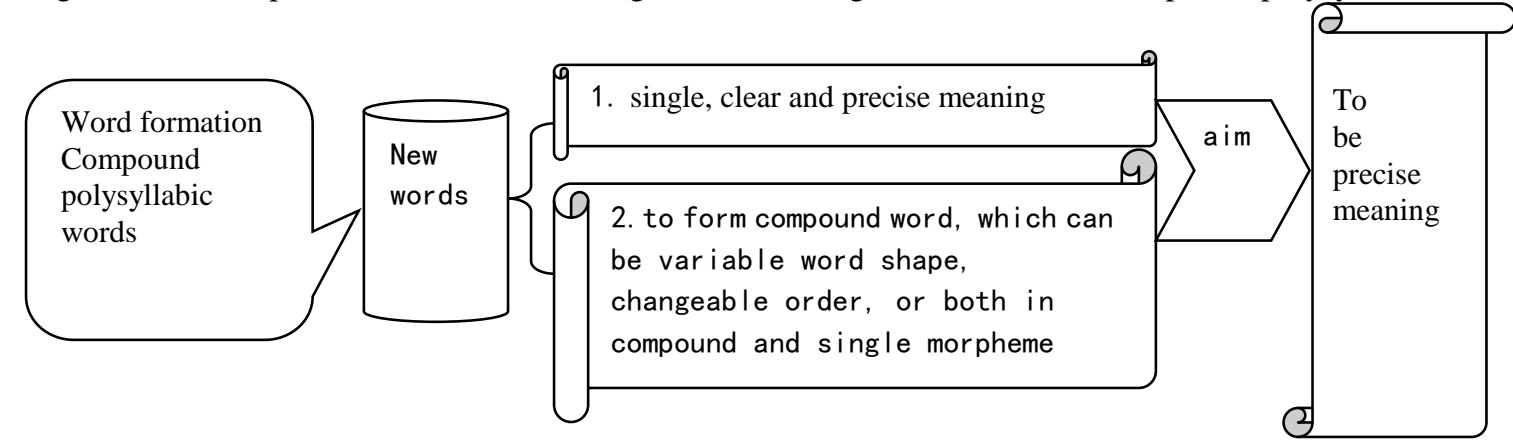




\section{Conclusions}

In summary, from the etymology features, Gan dialect vocabulary illustrates the inherent rule on the combination of native pronunciation and meaning and the conventional social members, also the development of the derived new words from the etymology of words. Lots of new words fundamentally depend on the composition of the polysyllabic words, whi-ch is the inevitable result of subjective and objective requirements.

To investigate the etymology features of the vocabulary in Gan dialects has a practical and positive significance for people to explore the emergence and development of the Gan dialect and other related issues.

\section{References}

[1] Wang Li. On Etymology Words[J]. Chinese Language, 1978 (1).

[2] Zeng Zhaocong. Research Status and Prospect of Chinese Etymology[J]. Journal of Jinan University (PHILOSOPHY AND SOCIAL SCIENCES), 2003 (4).

[3] Liu Zhenqian, Zhuang Huibin. Etymology Discrimination on Chinese Character "jiang" -Discussion with Mr. Zhang Hongming[J]. Journal of Shandong University (PHILOSOPHY AND SOCIAL SCIENCES EDITION), 2011 (5).

[4] Wang Mingyu. Study on the Etymology of "tianzhu" [J]. Linguistic Researches, 2012 (3).

[5] Chen Wenjie. The source of the Etymology of the Buddha[J]. Journal of Wuhan University (HUMANITIES SCIENCE EDITION), 2013 (4).

[6] Wang Ning, A few questions about Chinese Etymology[J]. Journal of Shaanxi Normal University, $2001(1)$.

[7] Ye Feisheng, Xu Tongqiang. Linguistics for Learners[M]. Rvised by Wang Hongjun, Li Juan. Beijing: Peking University press, 2010

[8] Shao Baiming, History of Nanchang Dialect Vocabulary[J]. Jiangxi Social Sciences, 2003 (6).

[9] Wang Li. Homology Dictionary [M]. Beijing: the Commercial Press, 1982

[10] Yu Liming, Tan Dailong. Diachronic Analysis in Synchronic Materials - Study on the Development of Chinese Vocabulary[J]. Journal of Sichuan University, 2004 (5).

[11] Wang Li. History of Chinese Language[M]. Beijing: Zhonghua Book Company, 1980

[12] Tang Yuming. Study on Jinwen Polysyllabic Words -- the Disyllablization of Chinese Origin[A], Anthology of Famous Middle-aged Linguists (Tang Yuming volume) [C]. Hefei: Anhui Education Press, 2002

[13] Xiang Xi. Brief History of Chinese [M]. Beijing: Higher Education Press, 1993 